\title{
LA ANTIGUEDAD DEL HOMBRE EN EL NORDESTE DE BRASIL
}

Gabriela Martin*

MARTIN, G. La antiguedad del Hombre en el nordeste de Brasil. Rev. do Museu de Arqueologia e Etnologia, S. Paulo, 2:7-12, 1992.

RESUMO: Entre os pré-historiadores americanistas existem várias tendências em relação à antiguidade do povoamento americano. As mais conservadoras não aceitam o povoamento do continente em datas anteriores a 20.000 anos e as mais avançadas chegam a admitir a possibilidade do homo erectus ter chegado à América 200 ou 300 mil anos atrás. Neste artigo resume-se os dados cronológicos conhecidos em relação a esse tema em comparação aos dados relativos ao Nordeste do Brasil. \section{Datação.}

UNITERMOS: Arqueologia americana. Povoamento da América.

El conocido americanista argentino Juan Schobinger publicó un interesante artículo titulado "200.000 años del hombre en América: ¿Que pensar?", en el homenaje ofrecido a E. Ripoll-Perelló con motivo de su jubilación como Director del Museo Arqueológico Nacional, de Madrid. El trabajo comienza recordando la pregunta ¿Que Pensar? formulada por Ripoll-Perelló comentando un articulo de Henry de Lumley, publicado en L'Anthropologie, en 1987, en el que comunicaba el hallazgo de instrumentos tallados asociados a fauna del Pleistoceno Medio en el Nordeste de Brasil, durante las excavaciones arqueológicas del abrigo Toca da Esperança, en Central, Estado de Bahia. Consistía en piezas liticas toscas, realizadas, supuestamente, con anterioridad al último periodo interglaciar de Sangamon. Estas excavaciones fueron realizadas en 1986 y dirijidas por Maria C. Beltrão del Museo Nacional de Rio de Janeiro, en colaboración con otros especialistas franceses y

(*) Universidade Federal de Pernambuco. canadienses. Para determinar las fechas se realizó el método del desequilibrio de la serie del Uranio sobre huesos de megafauna, realizado por Yuji Yokoyama en el Laboratoire des Faibles Raioactivités (CNRS).

En su artículo, el Prof. Schobinger, con la autoridad que le da su basto conocimiento de la Prehistoria Americana, se deshace de las ideas preconcebidas que pretende imponer la ortodoxia de los prehistoriadores americanos y analiza a fondo las posiblidades de que el homo erectus haya llegado al continente americano, miles años antes de que lo hiciera el homo sapiens sapiens. El citado autor recoge también dados de cuatro yacimientos norteamericanos, en los que la presencia humana podria fecharse entre 100 y 300 mil años, que formarian, posiblemente, el conjunto de yacimientos prehistóricos mas antiguos de América, a saber: Old Crow, en la cuenca del Yukon, NO de Canadá (con los resultados publicados por William Irving y su equipo); Texas Street, en el valle del Mision River en el Sur de California (trabajos publicados por el canadiense Bryan Reeves em 1986) y Calico 
Mountains en el SE de California (Excavaciones realizads por Ruth Simpson, directora del Museo de San Bernardino). Las industrias líticas de este último conjunto son basicamente choppers y hachas de mano similares a las de Chou-ku-tien. El yacimiento de Calico fué fechado geologicamente y por análisis de uranio-torio sobre las pátinas de carbonato de cálcio, que cubrian muchas de las piezas y que proporcionaron cronologias entre 150 y 200 mil años. ${ }^{1}$

Otro conjunto prehistórico con cronologías semejantes, es el de Valsequillo en el estado Mexicano de Puebla, con fechas obtenidas por el mismo método U-Th sobre huesos y por potasio-argonio sobre cenizas volcánicas del yacimiento de Huyatlaco, escavado por Irving-Williams y Steen McIntyre.

Las bajas cronologias de los yacimientos citados han sido objeto de criticas severas por parte del stablishment cientifico, desde los que prefieren simplesmente no darse por enterados hasta los que recurren al sarcasmo y hasta a la mala fé. No pretendemos discutir aquí las polémicas que este tema ha suscitado, solamente queremos presentar los hechos poniendo especial atención en los datos que tenemos, en la actualidad, en el NE de Brasil.

Hay otro bloque de datos e hipótesis sobre la antiguedad del hombre en América que la situa entre los 25 a los 50 mil años y es tan discutido por la americanistica tradicional como las fechas del grupo anterior, posición conservadora mantenida especialmente por el Bureau of American Ethnology de la Smithsonian Institution de Washington, institución que continua todavia la tradición crítica de Ales Hrdlicka, su antiguo director. Esta linea intransigente en contra de una antiguedad mayor a los 12.000 años, la mantienen, en la actualidad, Thomas Lynch, de la Universidad de Corenell y Dena Dincauze de la Universidad de Massachusetts, cuya posición se ha visto apoyada, no pocas veces, por la incredulidad de los prehistoriadores europeos. En el citado periodo cronológico se situan varios yacimientos del Sur de Estados Unidos y de México, además del yacimiento El bosque en

(1) Isla de Santa Rosa y China Lake en California; Levi y Lewisville en Texas; Isla de Avery en Luisiana y los yacimientos del area de Valsequillo, en México. el NO de Nicaragua. En el continente sudamericano está Monte Verde en el Sur de Chile, con cronología de 33.000 años del que ya se habian obtenido anteriormente fechas en torno de los 13.000 años BP. Se ha dado una fecha de 43.000 años, todavía dudosa, en Coribe, SO de Bahia (Brasil). Decimos dudosa, aunque se trata de una estructura en forma de fogón en la que se quemaron moluscos, no se encontraron instrumentos líticos. Por último, el ya internacionalmente famoso abrigo de Boqueirão da Pedra Furada en São Raimundo Nonato, Estado de Piauí en el NE de Brasil, con cronologías pleistocénicas de 20 a $48 \mathrm{mil}$ años y holocénicas de 6 a 10 mil años BP, es el mas importante yacimiento, dentro del tema que tratamos. Las excavaciones de este abrigo se prolongaron durante diez años dirigidas por Niède Guidón del CNRS (Paris) y Directora del Museo del Hombre Americano en Brasil. De los distintos niveles de la excavación que se completó en 1988, se obtuvieron 51 fechas de Carbono-14, de las que 16 corresponden al Holoceno y las restantes al Pleistoceno. La mas antigua es de 48000 años BP, obtenida con acelerador de massa y la mas moderna de 6160 BP. Por debajo del nivel mas antiguo fechado, existen todavía restos de ocupación humana de un metro de espesor. El que se haya ocupado este abrigo por largos periodos de tiempo, se explica por su situación previlegiada en medio de una región semidesiértica. Está formado por un enorme paredón inclinado, de mas de treinta metros de altura, que forma un abrigo siempre umbrio en una región extremadamente cálida, con temperaturas diurnas de hasta $40^{\circ} \mathrm{C}$. Además, en el lado izquierdo del mismo, existe un gran depósito natural excavado en la roca que puede almacenar 12000 litros de agua de lluvia.

La Pedra Furada fué sin duda un importante centro ceremonial en la Prehistoria. Sus paredes están cubiertas de bellas pinturas rupestres policrómicas figurativas. En la actualidad ha sido transformado en un Museo al aire libre, en el que sea posible contemplar las pinturas desde una pasarela, asi como la formación estratigráfica del yacimiento de la que se ha dejado un testigo de la secuencia.

El tercer bloque de yacimientos lo forma un grupo del Pleistoceno Final y de comienzos del Holoceno, con cronologias entre 10 y 20 mil años, que reunen un número considerable 
MARTIN, G. La antiguedad del Hombre en el nordeste de Brasil. Rev. do Museu de Arqueologia e Eınologia, S. Paulo, 2:7-12, 1992.

de abrigos y campamentos al aire libre en toda América, desde el Canadá hasta la Patagonia. Estas fechas han sido aceptadas por las escuelas mas conservadoras aunque todavía queden algumas reservas si se trata de América del Sur y si las fechas se aproximan a los $20 \mathrm{mil}$ años. En Brasil y dentro de estos limites cronológicos están los yacimientos de Lapa Vermelha IV, en Minas Gerais (excavado por A. Laming-Emperaire con cronologías que llegan hasta los 20 mil años), Alice Boer, en São Paulo, también con 20 mil años (excavaciones realizadas por M.C. Beltrão y J. Danon), en Serranópolis al Sur de Goiás, con fechas a partir de 13000 anos (resultado de las investigaciones de P. I. Schmitz), Lapa do Boquete en el valle de Peruaçú (Minas Gerais), donde A. Prous obtuvo una fecha de 11000 años BP.

En el nordeste de Brasil se obtuvieron fechas seguras radiocarbónicas por encima o alrededor de los 10000 años en los yacimientos de Coribe y Central, Estado de Bahia (abrigos de la Toca dos Buzios y Toca de Manoel Latão); en el Estado de Pernambuco, en Bom Jar$\operatorname{dim}$ (Chã do Caboclo) y en Brejo da Madre de Deus (Furna do Estrago). En el Estado de Rio Grande del Norte, ${ }^{2}$ hay también una fecha de 9410 años BP procedente del abrigo Mirador, cerca del pueblo de Parelhas y mas dos fechas, obtenidas recientemente, del abrigo Sitio do Alexandre, en Carnaúba dos Dantas, de 9400 e 8280 años BP. En el Estado de Piaui existe el magnifico conjunto arqueológico de São Raimundo Nonato, donde además del $\mathrm{Bo}$ queirão da Pedra Furada ya citado, se han logrado cronologias en torno de 10-15 mil años en los abrigos Sítios do Meio (12-14 mil años BP) y Toca do Baixão do Perna I (com diez fechas de C-14 comprendidas entre 3800 años BP); Toca da Serra do Bojo con cuatro fechas entre 7000 y $9700 \mathrm{BP}$. Otros abrigos situados también en el area arqueológica del SE de Piauí han proporcionado varias fechas 7000 y 9700 años BP. Finalmente, en el valle de San Francisco, cerca del pueblo de Petrolândia, en el

(2) Las fechas de Rio Grande del Norte y las de la Gruta do Padre y el Letreiro do Sobrado, en Pernambuco, se obtuvieron en el Laboratorio de Geocronologia del Instituto de Fisica-Quimica "Rocasolano", en el Consejo de Investigaciones Cientíticas de Madrid y a cuyo director, Dr. Fernan Alonso, queremos agradecer su colaboración con la Arqueologia Brasileña.
Estado de Pernambuco, la Gruta do Padre y el abrigo Letreiro do Sobarado, proporcionaron cronologias entre 5 y 7 mil años BP.

Muchos investigadores se resisten a aceptar la cronologia de alguno o de los tres grupos en que hemos dividido las fechas para la Prehistória de América, debido a que, además de lo poco convincente de algunos de los datos presentados, existe el prejuicio de que en el Nuevo Mundo todo deberia ser mas "nuevo" que en el Viejo según la teoria tradicional de que los primeiros pobladores de América ya eran cazadores especializados con tecnologia litica de tipo Sandia, Clovis, Folsom y Yuma. Esto ha llevado a no aceptar la existencia de un Paleolítico Americano con fechas muy antiguas y hay grandes desacuerdos a la hora de utilizar las terminologías procurando apartarse de los modelos europeos, posición critica de la que tampoco se han librado varios investigadores brasileños. Como las pruebas son cada vez mas evidentes, la tendencia actual es no aceptar fechas mas antiguas en América del Sur que en el Norte, defendiendola con el argumento de la via única de Bering. La población múltiple en épocas pleistocéncias muy antiguas, se acepta poco y apenas se discute, apesar de que ya existe un movimiento entre algunos americanistas que vuelve a plantear la discusión en torno a los posibles vias atlánticas, hipótesis levantada por J. Alcina Franch en la década de 50 y abandonada en los años 70. La teoria de una via atlántica arcaica para el poblamiento del continente sudamericano, cuenta principalmente en la difusión del $A n$ cylostoma duodenale, parásito humano de origem africana y asiática que no suporta bajas temperaturas y cuya presencia fué detectada en el SE de Piaui, en coprolitos humanos fechados en torno de 7000 anõs BP, trabajo realizado por Adauto de Araújo, da FIOCRUZ.

Antes de los años 50, investigadores no comprometidos con la ortodoxia de la tradición hrdlickariana, comenzaron a admitir la posibilidad de que el poblamiento de América fuese mucho mas antiguo y por vias múltiples. No cabe aqui dedicar mucho espacio a la bibliografía sobre esta polémica ya harto conocida, pero merecen recordarse los trabajos de Bosch Gimpera, Canals Frau, Paul Rivet, Menghin y mas tarde Gordon Willey, que en su conocida obra An Introduction to American Archaeology (1966-71), esboza la existencia 
MARTIN, G. La antiguedad del Hombre en el nordeste de Brasil. Rev. do Museu de Arqueologia e Etnologia, S. Paulo, 2:7-12, 1992.

de un Early Lithic Stage por el que no dejó de ser criticado.

Vemos que los tres grupos cronológicos que se discuten aparecen en el Nordeste de Brasil, lo que resulta sugestivo si tenemos en cuenta que se trata de una inmensa región de un millón y medio de $\mathrm{km}^{2}$, casi inexplorada arqueologicamente y de la que Sanders y Marino, em su Prehistoria del Nuevo Mundo, afirmaban, hace menos de 25 años, que seguramente nunca habia sido ocupada en la Prehistoria. Sin embargo, en todo los yacimientos incluídos cronologicamente en los dos grupos mas antiguos, falta el elemento clave que seria la presencia física del hombre, los restos fósiles que confirmen definitivamente la existencia humana en el NE de Brasil durante el Pleistoceno. Pero hasta el momento ningún hallazgo ha sido confirmado y el early man brasileño continua escondiendo su rostro. Los restos oseos humanos no se remontan a mas de diez mil años en todo el Brsil y el gran reto que se nos presenta es hallar restos humanos con cronologías antíguas confirmadas.

Maria C. Beltrão, con el gran entusiasmo que la caracteriza, está convencida de que tarde o temprano se encontrarán restos del homo erectus en América cuyos indicios podrian encontrarse en los abrigos de Central, en Bahia. En esta región se encontraron dos craneos de tipo "Lagoa Santa" pero no se han fechado. Se considera que este grupo tiene una cronología en torno de los 10000 años.

La pionera de la investigación en Brasil del que hemos llamado "segundo bloque cronológico americano", Niède Guidón, espera también el descubrimiento del hombre pleistocénico del Nordeste, para lo que ha intensificado sus trabajos en las grutas calcareas del SO del Piauí, área central de sus investigaciones.

Se multiplican poco a poco, en el NE de Brasil, las fechas de $\mathrm{C}-14$ correspondientes al final del Pleistoceno y de los comienzos del Holoceno, pero los restos oseos humanos son escasos o pertenecen a periodos más recientes. Un hallazgo importante que debemos a la $\mathrm{Dr}^{2}$ Guidón, es el de un esqueleto de la gruta Sítio do Antoniāo, también en el SE de Piauí (São Raimundo Nonato), fechado en 9500 años BP y que sería el resto humano mas antiguo del NE de Brasil encontrado hasta ahora. Aparte de este esqueleto, tenemos la fecha de 9410 años BP para los enterramientos infantiles del abrigo Mirador, en Rio Grande del Norte al que ya nos hemos referido, pero esta fecha se obtuvo del carbón de la hoguera utilizada en la incineración de los cadáveres, mientras que la fecha del Piaui fué consecuencia del análisis de los proprios huesos.

Actualmente se está escavando el abrigo Sítio do Alexandre en Carnaúba dos Dantas, también en el Estado de Rio Grande del Norte, abrigo próximo al de Mirador y que presenta el mismo tipo de pinturas rupestres que el anterior. Se trata de una necrópolis indigena prehistórica de la que ya se han retirado restos de 23 esqueletos, cuando solamente se ha excavado un tercio del yacimiento. Se han señalado dos ocupaciones, diferenciadas por enterramientos primarios y secundarios y se han obtenido, hasta el momento de redactar estas lineas, cinco fechas radiocarbónicas con las que se han podido fechar tres esqueletos de 9400 años BP (un niño de pocos meses), 8280 años BP (enterramiento primario de un adulto) y 4710 años BP (enterramiento secudario de un adulto). El ajuar funerario recogido (collares de hueso y conchas, principalmente) es semejante al coletado en el abrigo Mirador, que proporcionó la fecha citada de 9410 años.

Los dos mayores conjuntos de restos humanos prehistóricos hallados en el Nordeste de Brasil, son mucho mas recientes, entre 2000 y 1000 años BP, excavados en la Gruta do Padre y en la Furna do Estrago, ambos en el Estado de Pernambuco. Las fechas de 70005000 para la primera y $11000-9000$ para la segunda, corresponden a ocupaciones anteriores a los enterramientos. Ha sido descubierto también, recientemente, un importante yacimiento en Canindé, en el Estado de Sergipe, en el área donde se construye la hidroeléctrica de Xingó, en el valle del rio San Francisco. El hallazgo se debe a la joven arqueóloga Cleonice Vergne de la Universidade Federal de Sergipe y consiste en una necrópolis indigena situada en una terraza del rio y de la que se han retirado 93 esqueletos. Hasta el momento, se han obtenido fechas radiocarbónicas entre 1300 y 3300 años BP.

Hacemos nuestro el consejo de J. Schobinger en su conocida Prehistoria de Sudamérica: "Hay que esperar". Pero hay que esperar sin un cómodo escepticismo que puede volverse anticientífico. Del mismo modo que 
MARTIN, G. La antiguedad del Hombre en el nordeste de Brasil. Rev. do Museu de Arqueologia e Etnologia, S. Paulo, 2:7-12, 1992.

el entusiasmo exagerado puede llevar a ver lo que no existe, también el escepticismo cerril pude llevar a no ver lo que realmente existe, si el arqueólogo se aferra a viejas teorias sin querer modificarlas, solamente por haberlas defendido durante décadas, aunque ya las evidencias sean otras. Es preciso esperar nuevas pruebas siempre con la mente abierta a cualquier teoría nueva que venga a confirmarse. Es también válido preguntarse $i$ $\mathrm{Y}$ porque no? Si hoy sabemos que decendientes del hombre de Chou-Ku-Tien o emparentados con el sinántropo, se adaptaron a climas siberianos extremadamente frios, nada impide que, si conocian la forma de conseguir fuego, atravesaran la Beringia antes del último interglaciar. También el caso de los australianos que navegaban grandes distancias se recuerda frecuentemente, cuando se quiere defender la llegada a América por vias oceánicas en épocas pleistocénicas. Una teoria interesante pero todavía en el terreno de la conjectura, sería que se los antepasados del homo sapiens consiguieron llegar a América, pueden haber sido pequeños grupos que se extinguieron, dando lugar al largo paréntesis que precedió a la llegada de otras oleadas en torno de 50000 años. Estos nuevos grupos dispersados por diferentes regiones del continente, pueden haberse extinguido también, lo que explicaría los largos periodos sin ocupación que se observan en algunos yacimientos que podriamos llamar estratégicos y que indican hiatos sin ocupación de 10 a 20 mil años. En este caso estaria el Boqueirão da Pedra Furada, en el SE de Piauí, para citar el mas importante yacimiento arqueológico del NE de Brasil, en cuanto a secuencia cronológica.

Por los datos que hoy se conocen, no se puede negar ya que el nordeste de Brasil estaba poblado por seres humanos hace por lo menos 50000 años, hombres que, sin puntas de proyectil especializadas, con instrumentos líticos toscos, utilzando puntas de madera y trampas, cazaban mastodontes, perezosos gigantes, caballos, llamas y otros animales menores, entre los que se cuentan gran número de roedores y de aves. Mayores evidencias de su presencia es solo cuestión de tiempo.

MARTIN, G. Early man in northeastern Brazil. Rev. do Museu de Arqueologia e Etnologia, S. Paulo, 2:7-12, 1992.

ABSTRACT: Americanist pre-historians have manifested a variety of opinions regarding the arrival of early man in the Americas. The most conservative do not accept the appearance of early man before $\mathbf{2 0 . 0 0 0}$ years ago, while others admit to a dating of 200.000-300.000 years. This article summarizes and compares the known data regarding the chronology of early man in the Americas with data relative to the Brazilian Northeast.

UNITERMS: American archaeology. Early man in America. Dating.

\section{Referencias bibliográficas}

ALVIM, M.C.; SOUZA, S.M.F. Os esqueletos humanos da Furna do Estrago, Brejo da Madre de Deus, PE. CLIO - Série Arqueológica, UFPE, Recife, 1:95-98, 1984.

BELTRĀO, M.C.; DANON, J.; ZULETA, E.; POUPEAU, G. Thermoluminiscence Dating of Burnt
Cherts From the Alice Böer Site (Brazil). A. L. Bryan (Ed.). New Evidence for the Pleistocene Peopling of The America. Center for the Study of Early Man. University of Maine at Orono, Maine, 1986: 203-213.

BELTRẢO, M.C.; DANON, J.A.; DORIA, F.A. Da- 
MARTIN, G. La antiguedad del Hombre en el nordeste de Brasil. Rev. do Museu de Arqueologia e Etnologia, S. Paulo, 2:7-12, 1992.

taçōes absolutas mais antigas para a presença humana na América. Ed. UFRJ, Rio de Janeiro, 1988: $1-49$.

GUIDON, N. Las unidades culturales de São Raimundo Nonato, Sudeste del Estado de Piaui, Brasil. A.L. Bryan (Ed.) New Evidence for the Pleistocene Peopling of the America. Center for the Study of Early Man, University of Maine at Orono, Maine, 1986:157-171.

- A sequêencia cultural da área de São Raimundo Nonato, Piauí. CLIO - Série Arqueológica, UFPE, Recife, 3: 137-144, 1986.

LAROCHE, A.G.; LAROCHE, A. Considerações sobre a Prehistória do Nordeste brasileiro nos tempos finais do Pleistoceno e início do Holoceno. Anais do I Simpósio de Préhistória do Nordeste Brasileiro. CLIO - Série Arqueológica, Extra, UFPE, Recife, 4:31-35, 1991.

LIMA, J.M.D. Arqueologia na Furna do Estrago, Brejo da Madre de Deus, PE. CLIO - Série Arqueológica, UFPE, Recife, 2: 97-112, 1985.

LUMLEY, H. de et al. Presence d'outils taillés associés à une faune quaternaire datée du Pleistocene Moyen dans La Toca da Esperança. Región de Central. Etat de Bahia, Brésil. L'Anthropologie, 91(4): 917-942, 1987.

—. Découverte d'outils taillés associés à des faunes du Pleistocene Moyen dans La Toca da Esperança. Etat de Bahia, Brésil. Compte Rendu Ácadémie de Sciences de Paris, 306, Serie II: 241-247, 1987.

MARTIN, G. Arte rupestre no Seridó (RN). O Sítio
Mirador no Boqueirāo de Parelhas. CLIO - Série Arqueológica, UFPE, Recife, 1: 81-95, 1985.

—. O adeus à Gruta do Padre, Petrolândia, PE. A Tradição Itaparica de coletores-caçadores no Médio São Francisco. CLIO - Série Arqueológica, UFPE, Recife, 6: 31-68, 1990.

—. Novos dados sobre as pinturas rupestres do Seridó, no Rio Grande do Norte. Anais do I Simpósio de Pré-história do Nordeste Brasileiro. CLIO - Série Arqueológica, Extra, UFPE, Recife, 4:137-141, 1991.

MARTIN, G.; ROCHA, J. O abrigo "Letreiro do Sobrado". Petrolândia, PE. Anais da IV Reunião Científica da Sociedade de Arqueologia Brasileira - SAB. Dédalo, São Paulo, Publicaçōes Avulsas, USP, $1:$ 473-486, 1989.

PROUS, A. Os mais antigos vestígios arqueológicos no Brasil Central, Estado de Minas Gerais, Goiás e Bahia. A. L. Bryan (Ed.). New Evidence for the Pleistocene Peopling of the America. Center for the Study of Early Man, University of Maine at Orono, Maine, 19866:173-181.

SCHMITZ, P.I. Cazadores Antiguos en el Sudeste de Goiás, Brasil. A.L. Bryan (Ed.). New Evidence for the Pleistocene Peopling of the America. Center for the Study of Early Man, University of Maine at Orono, Maine, 1986: 183-193.

SCHOBINGER, J. Prehistoria de Sudamérica. Culturas Precerámicas. Alianza Editorial, Madrid, 1988.

—. 200.000 años del Hombre en América: Que pensar? Espacio, Tiempo y Forma, Serie I, Prehistoria, UNED, Madrid, 1988. 